\title{
Forcing phagosomes to mature
}

The intracellular pathogen Mycobacterium tuberculosis persists in phagocytic cells by inhibiting maturation of the phagosome. Now, a study published in Cell shows that the induction of autophagy (an intracellular pathway that facilitates the turnover of cytoplasmic macromolecules and organelles) can overcome this block in phagosomal maturation and that the antimycobacterial effects of interferon- $\gamma($ IFN- $\gamma$ ) can, in part, be explained by its ability to stimulate autophagy.

M. tuberculosis-mediated inhibition of phagosomal maturation is associated with decreased phagosome acidification, which results from mycobacterial disruption of intracellular-trafficking pathways that depend on the phosphatidylinositol 3-kinase VPS34. As VPS34 is also crucial for autophagy, Gutierrez et al. set out to investigate whether inducing autophagy would overcome the block in maturation of mycobacterial phagosomes. Exposure of Mycobacterium bovis bacillus Calmette-Guérin (BCG)-infected macrophages (using the mouse macrophage cell line RAW 264.7) to two autophagy stimuli — starvation conditions or rapamycin (a pharmacological inhibitor of MTOR, the serine/threonine kinase target of rapamycin) - led to increased acidification of mycobacterial phagosomes. Further evidence that mycobacterial phagosomes had matured and were destined for the autophagy pathway was provided by the observation that starvation resulted in co-localization of mycobacterial phagosomes with lateendosomal/lysosomal markers, including cathepsin D and LAMP1, and proteins involved in autophagy, such as LC3 and beclin-1.

The induction of autophagy in RAW 264.7 cells infected with BCG was associated with a decrease in mycobacterial survival. Similar inhibition of mycobacterial survival was observed when primary human and mouse macrophages infected with
BCG, or RAW 264.7 cells infected with the virulent $M$. tuberculosis H37Rv, were induced to undergo autophagy.

IFN- $\gamma$ is a crucial effector of the antimycobacterial immune response, and it was shown to induce autophagy in RAW 264.7 cells and to induce partial co-localization of the autophagosomal marker LC3 with mycobacterial phagosomes. Furthermore, overexpression of LRG47, the recently described downstream effector of IFN- $\gamma$, caused increased maturation of mycobacterial phagosomes.

This study shows that the induction of autophagy promotes maturation of mycobacterial phagosomes, which thereby decreases mycobacterial survival. It also shows that IFN- $\gamma$ induces autophagy and that LRG47 is probably an important effector of this antimycobacterial effect of IFN- $\gamma$. On the basis of these results, the authors suggest that autophagy is part of the innate immune response to intracellular pathogens.

Karen Honey

(0) References and links ORIGINAL RESEARCH PAPER Gutierrez, M. G. et al. Autophagy is a defense mechanism inhibiting $B C G$ and Mycobacterium tuberculosis survival in infected macrophages. Cell 18 Nov 2004 (doi:10.1016/S0092867404011067).

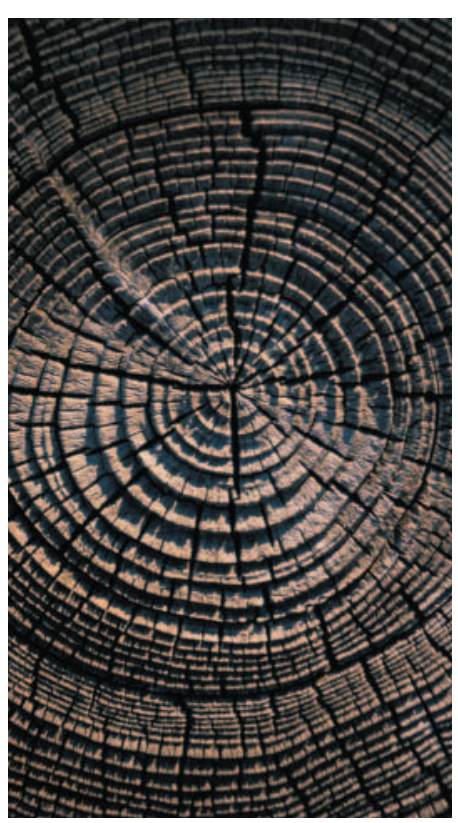

\section{IN BRIEF}

LYMPHOCYTE MIGRATION

CLEVER-1 mediates lymphocyte transmigration through vascular and lymphatic endothelium.

Salmi, M. et al. Blood 78, 3849-3857 (2004).

Lymphocyte trafficking from the blood to the tissues has been extensively studied and is described by the multistep adhesion cascade. By contrast, the mechanism used by lymphocytes to cross lymphatic-vessel walls is much less well understood. Salmi and colleagues show that the glycoprotein CLEVER1 (common lymphatic endothelial and vascular endothelial receptor 1) is constitutively expressed on lymphatic endothelial vessels, and its expression is upregulated by inflamed vascular endothelial cells. CLEVER1 can mediate the adhesion and migration of lymphocytes (and the adhesion of tumour cells) through lymphatic-vessel walls, as well as through blood-vessel walls, under physiologically relevant shear forces. CLEVER1 is therefore a potential target for the development of new therapies for inflammation and cancer.

\section{HIV}

Secretory leukocyte protease inhibitor binds to annexin II, a cofactor for macrophage HIV-1 infection.

Ma, G. et al. J. Exp. Med. 200, 1337-1346 (2004).

One of the earliest identified functions of the serine-protease inhibitor SLPI (secretory leukocyte protease inhibitor) was as an inhibitor of HIV-1 infection of macrophages. However, the identity of the cell-surface receptor for SLPI is unknown. This study shows that the phospholipid-binding protein annexin II is a receptor for SLPI. Inhibitors of annexin II mimicked the kinetics of action of HIV-1 suppression by SLPI, indicating a connection. The authors identified annexin II as a fusogenic cofactor for HIV-1 infection and a possible target for new therapeutics: HIV-1 can bind annexin II through phosphatidylserine molecules in the HIV-1 envelope, which are acquired from the host during viral exit from infected cells.

\section{MACROPHAGES}

IFN regulatory factor 3-dependent induction of type I IFNs by intracellular bacteria is mediated by a TLR- and Nod2-independent mechanism.

Stockinger, S. et al. J. Immunol. 173, 7416-7425 (2004).

Type I interferons (IFNs) produced by macrophages in response to infection with Listeria monocytogenes sensitize macrophages to L. monocytogenes-induced cell death. In this study, Stockinger et al. sought to determine the molecular mechanisms of the L. monocytogenes-mediated induction of genes that encode type I IFNs. IFN- $\alpha$ production by $L$. monocytogenes-infected macrophages was dependent on IFN- $\beta$ production, and IFN- $\beta$ production was dependent on the presence of IFN-regulatory factor 3 (IRF3). IFN- $\beta$ production was not affected by the absence of the pathogen-recognition receptors Toll-like receptor 4 (TLR4), TLR9 or nucleotide-binding oligomerization domain protein 2 (NOD2), or of the adaptor molecules MyD88, TRIF or TRAF. So, the authors suggest that L. monocytogenes targets IRF3 through a new pathway, which is TLR and NOD2 independent. 